\title{
Breve análisis del proyecto educativo "Deportes del buen vivir" aplicado en la unidad educativa Liceo Naval de Guayaquil periodos lectivos 2014 -2015, 2015 $-2016$
}

\section{Brief analysis of the educational project "Sports of good living" applied in the Liceo Naval educational unit of Guayaquil, 2014-2015, 2015-2016}

Lic. David Morales Neira MSc,

Universidad Internacional del Ecuador, Ecuador

Lic. Alberto Saltos Aldaz

Prof. Roberto Rojas Bajaña

Unidad Educativa Liceo Naval de Guayaquil, Ecuador

Lic. Marcos Murillo Salazar MSc

Universidad Católica de Santiago de Guayaquil, Ecuador

Autor para correspondencia: jimurarobert27@hotmail.com, saltosalberto@ hotmail.com , damoralesne@internacional.edu.ec, jobmorales87@hotmail.com, saltosalberto@ hotmail.com, margena12@hotmail.com

Fecha de recepción: 15 de Junio de 2016 - Fecha de aceptación: 28 de Junio de 2016

Resumen: En el presente trabajo de investigación muestra la aplicación del Proyecto de Innovación Educativa "Deportes del Buen Vivir" gracias al aumento de la carga horaria de 2 a 5 horas pedagógicas semanales de Educación Física en la malla curricular desde el Primero a Décimo año de Educación General Básica. Cuyo propósito es identificar la percepción del alumnado sobre el proceso enseñanza - aprendizaje mediante el análisis documental, observación y encuestas a los estudiantes de 8vo, 9no y 10mo de Educación Básica Superior matutina y vespertina. Se elevó el nivel de calidad en la adquisición de nuevos saberes motrices con una base educativa pre deportiva. El Proyecto guarda una íntima relación con el Currículo Nacional de Educación Física 2012, dando realce al empoderamiento de la práctica deportiva dentro y fuera de la institución. Los criterios que justifican este estudio es el cumplimiento de la Constitución de la República del Ecuador, Plan Nacional del Buen Vivir, Plan Decenal, Ley Orgánica de Educación Intercultural y su Reglamento, La Ley del Deporte, Educación Física y Recreación, Código de la Niñez y Adolescencia, entre otros.

Palabras claves: plan nacional del buen vivir; acuerdo ministerial 0041-14; proceso enseñañzaaprendizaje; alfabetización motriz; educación física de calidad

Abstract: In the present research work shows the application of Educational Innovation Project "Sports for Good Living" by increasing the number of hours of 2 to 5 teaching hours per week of physical education in the curriculum from first to tenth year of General Education Basic. Whose purpose is to identify the perception of students on teaching - learning process through document analysis, observation and student surveys of 8th, 9th and 10th of morning and evening Superior Basic Education. the quality level rose in the acquisition of new knowledge with a pre motor sports educational base. The project keeps a close relationship with the National Physical Education Curriculum 2012, giving prominence to the empowerment of sport within and outside the 
institution. The criteria that justify this study is the fulfillment of the Constitution of the Republic of Ecuador, National Plan for Good Living, Ten-Year Plan, Organic Law of Intercultural Education and its Regulations, the Law of Sport, Physical Education and Recreation, Code of Childhood and Adolescents, among others.

Key words: national plan for good living; ministerial agreement 0041-14; teaching and learning; literacy drive; quality physical education process

\section{Introducción}

E1 Proyecto es relevante debido que la Unidad Educativa Liceo Naval "Cmdte. Rafael Andrade Lalama" de la ciudad de Guayaquil, desde su fundación (20 de octubre de 1970), se ha caracterizado en masificar la práctica deportiva. La institución hasta el periodo escolar 2013 2014, ofertó la práctica deportiva como extracurricular, y el aumento de la carga horaria en Educación Física facilitó el ingreso dentro de la carga horaria el "Proyecto Deportes del Buen Vivir", en el cual se destinan 2 horas pedagógicas en las disciplinas deportivas a nivel semanal por curso: segundo, tercero, cuarto, quinto, sexto, séptimo, octavo, noveno y décimo año de educación general básica.

El Liceo Naval de Guayaquil como filosofía institucional, manifiesta que por medio de la educación y la cultura física favorece de manera directa a la adquisición de destrezas motoras, cognitivas y afectivas necesarias para aprovechar los conocimientos, interactuar con el mundo físico e integrarse plenamente al mundo social. En base a estos parámetros educativos y legales, el artículo evalúa “El Proyecto Deportes del Buen Vivir" aplicado en los periodos lectivo 20142015 y 2015 -2016, cursos: octavo, noveno y décimo año de Educación Básica Superior.

Una vez siendo oficializadas las 5 horas de Educación Física, los docentes: Alberto Saltos, Roberto Rojas y David Morales (2014) plantearon una propuesta innovadora para la distribución del talento humano y contenidos del currículo de EF vigente para el periodo lectivo 2014-2015, siendo reformado en el periodo lectivo 2015- 2016.

Para determinar los resultados de la percepción de los estudiantes de la Educación Básica Superior, jornadas matutina y vespertina, se utilizó el método descriptivo, estadístico y transversal en base a los resultados de los saberes de Lord.

\section{Marco teórico}

\section{Niveles de concreción curricular en el Ecuador.}

Según La Dirección Nacional de Currículo, (2016) establece 3 niveles de concreción:

El Ministerio de Educación, Ministerio del Deporte y La dirección Nacional de Currículo diseñaron el Macrocurrículo- Currículo de EF de 2012, vigente hasta inicios de 2016 (Marcelo Tovar, Roberto Rodríguez, 2012).

El segundo nivel, se especifica como plan Mesocurricular, fundamentado se basa en dos diseños específicos Plan Curricular Institucional (PCI) y el Programa Curricular Anual (PCA). 
El Tercer Nivel, Plan Microcurricular basado en el plan de clase o plan de destrezas.

Tabla 1. (Niveles de concreción en el Ecuador)

\begin{tabular}{|c|c|c|c|}
\hline Primer Nivel & Segundo & Nivel & Tercer Nivel \\
\hline -Macro & Meso & & Micro \\
\hline $\begin{array}{l}\text {-Ministerio de Educación } \\
\text {-Dirección Nacional de }\end{array}$ & -Instituciones educativas & & -Docentes \\
\hline Currículo & & & \\
\hline -Currículo Nacional & -Currículo institucional & & -Currículo de aula \\
\hline Obligatorio por asignatura & & & \\
\hline Prescriptivo & $\begin{array}{l}\text { Proyecto Educativo } \\
\text { Institucional (PEI) }\end{array}$ & $\begin{array}{c}\text { Plan Anual } \\
\text { Curricular (PCA) }\end{array}$ & $\begin{array}{l}\text {-Planificaciones de aula } \\
\text { Adaptaciones curriculares } \\
\text { (individuales y grupales) }\end{array}$ \\
\hline
\end{tabular}

Fuente: (Dirección Nacional de Currículo, 2016)

\section{Importancia de las actividades complementarias y extraescolares}

Las actividades complementarias y extracurriculares permiten la integración del niño(a), adolescente, permitiendo la formación de su "propia identidad". Se caracterizan por ser un recurso de interés educativo en la que resalta una metodología motivadora y lúdica para potenciar los aprendizajes adquiridos en las demás asignaturas.

Una educación basada en experiencias, por descubrimiento, vivencias con la naturaleza y su contexto a través de la acción motriz. Donde el estudiante va a manifestar sus intereses, deseos y aspiraciones por medio de la "observación, la exploración y la manipulación de la información" (Fernández, Análisis de las actividades complementarias y extraescolares y su valor educativo, 2015).

La UNESCO (1978) en el artículo 1 de la Carta de Internacional de la Educación Física y el Deporte, establece que su "acceso es un derecho fundamental para todos". Irina Bokova, Directora General de la UNESCO, la EF se basa sobre tres principios: "la igualdad, la protección y la participación significativa". La EF como herramienta para elevar el nivel de competencias en las otras áreas del conocimiento.

Según La UNESCO (2015) en la guía para los responsables políticos "Educación Física de calidad", afirma que la práctica de EF está disminuyendo en todas las regiones del mundo, considerándose una pandemia en altos números de sedentarismo generando enfermedades asociadas.

\section{Acuerdo Ministerial 0041-14, 11.}

Las horas pedagógicas en Educación Física según Espinosa (2014), oficializa la nueva malla curricular para Educación General Básica, la que muestra reajustes a la distribución de la carga horaria tomando la importancia de cada asignatura y actividad que permita el desarrollo integral de cada estudiante.

\section{Tabla 2. Carga horaria-Acuerdo Ministerial}


MALLA DE LA EDUCACIÓN GENERAL BÁSICA

\begin{tabular}{|c|c|c|c|c|c|c|c|c|c|c|}
\hline \multirow[b]{2}{*}{ ASIGNATURAS Y CLUBES } & \multicolumn{10}{|c|}{ GRADO } \\
\hline & 19 & $2=$ & $3^{2}$ & $4^{9}$ & $5^{2}$ & $6^{2}$ & $7^{9}$ & $8^{n}$ & 99 & $10^{\mathrm{e}}$ \\
\hline Lengua y Literatura & \multirow{5}{*}{25} & 12 & 12 & 9 & 9 & 9 & 9 & 6 & 6 & 6 \\
\hline Matemática & & 8 & 8 & 7 & 7 & 7 & 7 & 6 & 6 & 6 \\
\hline Entorno Natural y Social & & 5 & 5 & & & & & & & \\
\hline Ciencias Naturales & & & & 5 & 5 & 5 & 5 & 4 & 4 & 4 \\
\hline Estudios Sociales & & & & 4 & 4 & 4 & 4 & 4 & 4 & 4 \\
\hline Educación Estética & 2 & 2 & 2 & 2 & 2 & 2 & 2 & 2 & 2 & 2 \\
\hline Educación Física & 5 & 5 & 5 & 5 & 5 & 5 & 5 & 5 & 5 & 5 \\
\hline Lengua Extranjera & & & & & & & & 5 & 5 & 5 \\
\hline Clubes & 3 & 3 & 3 & 3 & 3 & 3 & 3 & 3 & 3 & 3 \\
\hline
\end{tabular}

Según La Secretaría Nacional de Planificación y Desarrollo (2013-2017), el Plan Nacional del Buen Vivir expresa en el objetivo 3, la finalidad de mejorar la calidad de vida de la población mediante el ejercicio pleno de los derechos del "Buen Vivir". En uno de los apartados "ocio, tiempo libre, deporte y actividad física", expresa que el deporte es un agente promotor de la calidad de vida de la población, ya que contribuye a la mejora de la salud, a la educación y a la organización comunitaria (p. 140).

La Constitución de la República del Ecuador, (2008) establece en el artículo 381 : “El Estado protegerá, promoverá y coordinará la Cultura Física que comprende el deporte, la educación física y la recreación”. La Ley del Deporte, Educación Física y Recreación, (2015) establece y regula las actividades para mejorar la condición de toda la población aporta a la consecución del Buen Vivir.

La Constitución de la República del Ecuador, (2008) en el Título VII, Régimen del Buen Vivir, Capítulo Primero Inclusión y equidad expresa:

"El Estado protegerá, promoverá y coordinará la Cultura Física que comprende el deporte, la educación física y la recreación, como actividades que contribuyen a la salud, formación y desarrollo integral de las personas; impulsará el acceso masivo al deporte y a las actividades deportivas a nivel formativo, barrial y parroquial; auspiciará la preparación y participación de los deportistas en competencias nacionales e internacionales, que incluyen los Juegos Olímpicos y Paraolímpicos; y fomentará la participación de las personas con discapacidad."

La Ley del Deporte, Educación Física y Recreación, (2010) en la Décimo Octava disposición general prescribe:

Deporte: El deporte es toda actividad física y mental caracterizada por una actitud lúcida y de afán competitivo de comprobación o desafío, dentro de disciplinas y normas preestablecidas constantes en los reglamentos de las organizaciones nacionales y/o internacionales correspondientes, orientadas a generar valores morales, cívicos y sociales y desarrollar fortalezas y habilidades susceptibles de potenciación. 
Educación Física: Es una disciplina que basa su accionar en la enseñanza y perfeccionamiento de movimientos corporales. Busca formar de una manera integral y armónica al ser humano, estimulando positivamente sus capacidades físicas.

Recreación: Son todas las actividades físicas lúdicas que empleen al tiempo libre de una manera planificada para constituirse en una verdadera terapia para el cuerpo y la mente, buscando un equilibrio biológico y social en la consecución de una mejor salud y calidad de vida.

\section{Metodología.}

\section{Distribución de las 5 horas de Educación Física semanales.}

Tabla 3. Distribución de las 5 horas de EF

\begin{tabular}{cc}
\hline Horas & Actividades \\
\hline 2 & Educación Física \\
2 & Deportes del Buen Vivir \\
1 & Recreación
\end{tabular}

Fuente : (Alberto Saltos, Roberto Rojas y David Morales, 2014-2015)

Elaborado por: Saltos, Morales \& Rojas.

\section{Personal docentes deportivos.}

En vista que el Departamento de Cultura Física del Liceo Naval de Guayaquil consta con 10 docentes especialistas en el área de La Educación Física y Deportes, distribuidos véase (tabla 4) con la finalidad de cumplir con las 30 horas clases dispuestas por el Ministerio de Educación del Ecuador.

Tabla 4. Disciplinas deportivas

\begin{tabular}{ccc}
\hline \multicolumn{2}{c}{ Disciplinas deportivas del "Buen Vivir" } & Género \\
\cline { 1 - 2 } Disciplina & $\mathrm{N}^{\circ}$ Docentes Deportivos & \\
Atletismo & 2 & 2 varones \\
Balonmamo & 1 & 1 varón \\
Fútbol & 3 & 3 varones \\
Natación & 4 & 3 varones, 1 dama \\
Total & 10 & 1 dama 9 varones \\
\hline Fuente: (Alberto Saltos, Roberto Rojas y David Morales, 2014-2015) \\
\multicolumn{2}{c}{ Elaborado por: Saltos, Morales \& Rojas. }
\end{tabular}

\section{Distribución en horarios del proyecto.}

Tabla 5. Horario de "Deportes del Buen Vivir"

\begin{tabular}{|c|c|c|c|c|c|c|}
\hline \multicolumn{7}{|c|}{ Jornada Matutina } \\
\hline Períodos & Horario & Lunes & Martes & Miércoles & Jueves & Viernes \\
\hline 1 & $\begin{array}{c}07 \mathrm{~h} 00 \\
07 \mathrm{~h} 40\end{array}$ & & & & & \\
\hline 2 & $\begin{array}{l}07 \mathrm{~h} 40 \\
08 \text { h } 20\end{array}$ & & & $\begin{array}{l}\text { 6to Básico } \\
\text { Escuela }\end{array}$ & & $\begin{array}{l}\text { 7to Básico } \\
\text { Escuela }\end{array}$ \\
\hline 3 & $\begin{array}{l}08 \text { h } 20 \\
09 \text { h } 00\end{array}$ & $\begin{array}{c}2 \text { do } \\
\text { Básico }\end{array}$ & $\begin{array}{l}3 \text { ro Básico } \\
\text { Escuela }\end{array}$ & & $\begin{array}{l}\text { 4to Básico } \\
\text { Escuela }\end{array}$ & \\
\hline
\end{tabular}




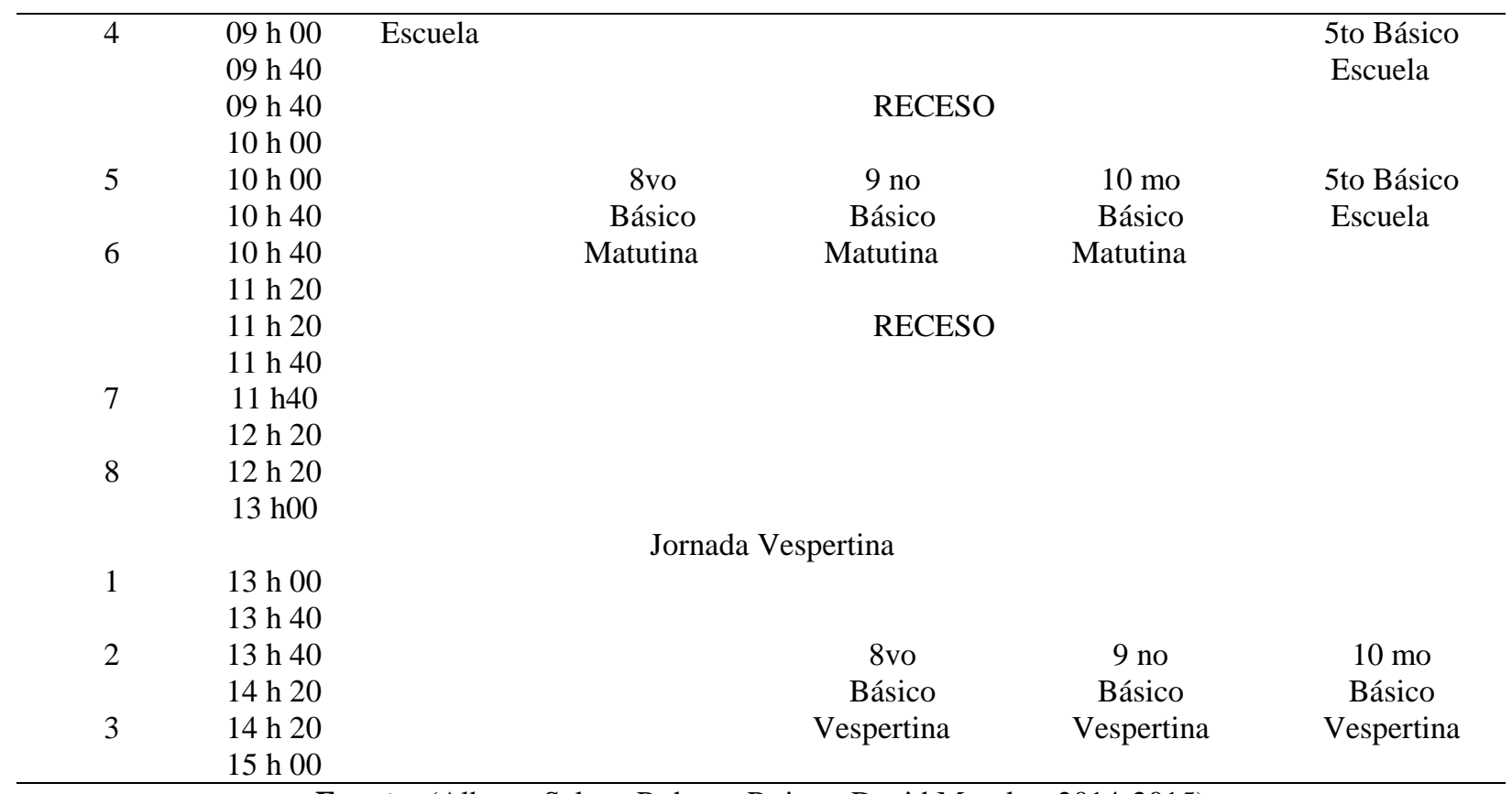

Fuente: (Alberto Saltos, Roberto Rojas y David Morales, 2014-2015)

Elaborado por: Saltos, Morales \& Rojas.

Según David Morales en el Trabajo de Fin de Máster, (2016), con la finalidad de identificar y analizar la percepción del alumnado en relación a la EF y el Proyecto "Deportes del Buen Vivir" aplicado en el octavo año de Educación Básica Superior, jornada vespertina. Se aplicó una encuesta el 27 de enero de 2016, a 35 estudiantes, 7 estudiantes por paralelo, de los 5 paralelos existentes: Alfa -Bravo-Charlie-Delta y Echo. Cuestionario de preguntas inéditas, validado por el Dr. Vicente Martínez de Haro, docente de la Universidad Autónoma de Madrid.

Constituido por 10 ítems o preguntas cerradas. El cuestionario presenta una breve introducción, informativo sobre: fines, objetivos y elementos que componen a la EF y porqué son importantes. Se aplicó la escala de alternativas: 1 a 4; 4 valor cualitativo "totalmente de acuerdo", 3 "de acuerdo", 2 "desacuerdo", 1 "totalmente en desacuerdo". Se aclara que no existen respuestas correctas o incorrectas, se establece la confidencialidad y privacidad de los datos informativos.

Se han distribuido los ítems de acuerdo al ámbito de los saberes de Delors (1996).

- Saber conocer las preguntas : : 1-2-6.

- Saber hacer las preguntas : $\quad$ : 3-4-5-10.

- Saber ser $\quad$ : 7-8.

- Saber vivir en comunidad la pregunta : 9.

La edad de los estudiantes se encuentra entre los 12 y 13 años. De los 35 encuestados se encuentra un equilibrio entre damas y varones.

Análisis.

El presente análisis se fundamenta en las tareas de investigación de los Postgradistas de La Universidad Autónoma de Madrid, de La Maestría en Formación de Profesorado, 
Especialidad Educación Física, promoción 2015-2016. A continuación, se van analizar los ítems agrupados en los 4 apartados mencionados anteriormente de manera cuantitativa.

\section{En relación al saber conocer de la $\mathbf{E F}$ :}

- El $89 \%$ están totalmente de acuerdo, el $11 \%$ de acuerdo, no hay respuestas desacuerdo y totalmente desacuerdo la EF es una asignatura que cumple con el desarrollo del Programa Nacional del Buen Vivir (pregunta 1).

- El $80 \%$ están totalmente de acuerdo, el $20 \%$ están de acuerdo, ningún estudiante manifestó en desacuerdo y totalmente en desacuerdo que el eje integrador de EF cumple con la realidad educativa (pregunta 2).

- El $74 \%$ están totalmente de acuerdo, el $26 \%$ de acuerdo, ningún estudiante manifestó en desacuerdo y totalmente en desacuerdo que la EF permite la manifestación del pensamiento crítico sobre las diferentes actividades y componentes del proceso enseñanza- aprendizaje (pregunta 6).

\section{En relación al saber hacer de la $\mathrm{EF}$ :}

- El $83 \%$ están totalmente de acuerdo, el $17 \%$ de acuerdo, ningún estudiante manifestó en desacuerdo y totalmente en desacuerdo que La EF propende la aplicación el uso productivo-deportivo del tiempo libre (pregunta 3).

- El $77 \%$ están totalmente de acuerdo, el $23 \%$ de acuerdo, ningún estudiante manifestó en desacuerdo y totalmente en desacuerdo que la EF permite aprender a resolver desafíos utilizando las herramientas adquiridas en el proceso educativo (pregunta 4).

- El $77 \%$ están totalmente de acuerdo, el $23 \%$ de acuerdo, ningún estudiante manifestó en desacuerdo y totalmente en desacuerdo que la EF dota de las herramientas para ser capaz de diseñar y aplicar un programa de salud (pregunta 5).

- El $89 \%$ están totalmente de acuerdo, el $11 \%$ de acuerdo, ningún estudiante manifestó en desacuerdo y totalmente en desacuerdo que la EF permite conocer y aplicar normas de seguridad en el uso de instalaciones deportivas (pregunta 10).

\section{En relación al saber ser de la $\mathrm{EF}$ :}

- El $74 \%$ están totalmente de acuerdo, el $26 \%$ de acuerdo, ningún estudiante manifestó en desacuerdo y totalmente en desacuerdo que la EF brinda la oportunidad de manifestar el pensamiento crítico sobre las diferentes actividades y componentes del proceso enseñanzaaprendizaje (pregunta 7).

- El $77 \%$ están totalmente de acuerdo, el $23 \%$ de acuerdo, ningún estudiante manifestó en desacuerdo y totalmente en desacuerdo que la EF faculta la participación en torneos internos-externos, son importantes en la formación de la conducta del estudiante y el trabajo cooperativo (pregunta 8).

\section{En relación al saber vivir en comunidad de la $\mathbf{E F}$ :}

- El $63 \%$ están totalmente de acuerdo, el $29 \%$ de acuerdo, el $6 \%$ en desacuerdo y el $3 \%$ totalmente en desacuerdo que la EF permite la sana convivencia con otras instituciones educativas (pregunta 9).

- El análisis colectivo según Delors (1996), haciendo énfasis en los saberes como pilares fundamentales en la formación del ser humano. Saber conocer, saber hacer, saber ser y saber vivir en comunidad. Se agruparon las preguntas o ítems: 
Tabla 6. Resumen del análisis de los cuestionarios por saberes

\begin{tabular}{lccc}
\hline \multicolumn{1}{c}{ Saberes } & Negación & Afirmación & Total \\
\hline Saber conocer: 1-2-6. & $0 \%$ & $100 \%$ & $100 \%$ \\
Saber hacer: 3-4-5-10. & $0 \%$ & $100 \%$ & $100 \%$ \\
Saber ser: 7-8. & $0 \%$ & $100 \%$ & $100 \%$ \\
Saber vivir: 9. & $9 \%$ & $91 \%$ & $100 \%$ \\
\hline
\end{tabular}

Fuente: Delors (1996).

Elaborado por: David Morales Neira

Según Roberto Rojas (2015), en la aplicación de la encuesta a 70 estudiantes de: 8- 9 y 10 mo año de Educación General Básica Superior, jornada Matutina del Liceo Naval de Guayaquil, cuyas características del cuestionario son: Preguntas cerradas bajo los parámetros: afirmación, negación e indiferente. Con el Aval del señor Vicerrector Académico y con el consentimiento del señor Rector del Plantel.

- Según los resultados de la pregunta 14 de los 70 encuestados el $100 \%$ manifiestan, sí les han servido las destrezas adquiridas dentro de las 5 horas de Educación Física en la vida cotidiana mientras que el $0 \%$ No dicen nada al respecto, y el $0 \%$ se muestra indiferente.

- Según los resultados de la pregunta 15 de los 70 encuestados el 95,7\% manifiestan, sí están de acuerdo con la distribución de las horas en: 2 Educación Física, 2 Deportes y 1 recreación dentro de las 5 horas de Educación Física mientras que el 4,3\% no, y el 0\% se muestra indiferente.

- Según los resultados de la pregunta 17 de los 70 encuestados el 100\% sí les gusta participar en los deportes dentro de las horas de Educación Física mientras que el 0\% No y el 0\% se muestra indiferente.

\section{Discusión}

- Se aplicó efectivamente el Proyecto de Innovación Educativa partiendo desde la planeación, socialización, organización y aplicación con los diferentes actores de la comunidad liceísta.

- Se puso de manifiesto el deficiente dominio curricular en el área de la defensa personal y la danza.

- Deficiente comunicación organizacional con las otras áreas en proyectos interdisciplinarios. Escasez en la toma de decisiones y la falta de disonancia cognitiva en el proceso educativo enfocado en el estudiante.

- En el saber conocer, El $81 \%$ de los cadetes, manifiestan la adquisición de nuevos aprendizajes cognitivos.

- En el saber hacer, el $82 \%$ de cadetes, indica la satisfacción de descubrir nuevas experiencias motrices pre deportivas.

- En el saber ser, el $76 \%$ de los cadetes, caracterizan a la EF, como asignatura que promueve valores.

- En el saber vivir en comunidad, el $63 \%$ de cadetes, afirman que la práctica deportiva fortalece el trabajo en equipo.

\section{Conclusión.}


El aumento de la carga horaria en Educación Física aumenta el compromiso de autoridades y cuerpo docente en fortalecer la alfabetización motriz, mejorar la calidad de la enseñanza, llevar una correcta aplicación de evaluaciones: diagnóstico, formativa y sumativa en el proceso educativo. Hacer uso de la autoevaluación, coevaluación y la heteroevaluación con la finalidad de mejorar la aplicabilidad del Currículo de Educación Física Vigente junto a proyectos de innovación educativa. El proyecto "Deportes del Buen Vivir" marca notablemente un caminoreferente en la distribución del talento humano. La oportunidad que goza el estudiante de practicar y competir dentro y fuera de la institución en múltiples disciplinas deportivas con docentes calificados.

\section{Bibliografía}

Acuerdo Ministerial 0041-143. (11 de marzo de 2014). educacion.gob.ec. Obtenido de http://educacion.gob.ec/wp-content/uploads/downloads/2014/03/ACUERDO-041-14.pdf

Alberto Saltos, David Morales, Roberto Rojas. (2014). Proyecto Deportes del Buen Vivir. Guayaquil, Guayas, Ecuador: Liceo Naval de Guayaquil.

Alberto Saltos, Roberto Rojas y David Morales. (2014-2015). Deportes del Buen Vivir. Guayaquil, Guayas, Ecuador: Unidad Educativa Liceo Naval "Cmdte. Rafael Andrade Lalama".

Asamblea Nacional Constituyente. (16 de marzo de 2016). Constitución de la República del Ecuador. Obtenido de http://www.asambleanacional.gov.ec/documentos/constitucion_de_bolsillo.pdf

Asamblea Nacional Constituyente. (16 de marzo de 2016). Ley del Deporte, Educación Física y Recreación. Obtenido de http://www.deporte.gob.ec/wpcontent/uploads/downloads/2015/03/Ley-del-Deporte.pdf

Asamblea Nacional Constituyente. (31 de marzo de 2011). Ley Orgánica de Educación Intercultural. $\begin{array}{lllll}\text { Recuperado el } 18 \text { de } & \text { de }\end{array}$ http://diccionario.administracionpublica.gob.ec/adjuntos/2loei.pdf

Carta Internacional de la Educación Física y el Deporte. (21 de noviembre de 1978). UNESCO. Obtenido de http://portal.unesco.org/: http://portal.unesco.org/es/ev.phpURL_ID=13150\&URL_DO=DO_TOPIC\&URL_SECTION=201.html

Constitución de la República del Ecuador. (2008). Constitución de la República del Ecuador. Obtenido de http://www.asambleanacional.gov.ec/documentos/constitucion_de_bolsillo.pdf

Correa, R. (jueves 26 de julio de 2012). Reglamento a Ley Orgánica de Educación Intercultural. Guayaquil: Mañosca No 201 y 10 de agosto.

Cultura Física Liceo Naval, D. D. (2015). Informe Anual 2014. Guayaquil.

David Morales, Alberto Saltos. (22 de octubre de 2015). Aprendizaje, enseñanza, innovación docente e iniciación a la investigación educativa en Educación Física. Análisis del Currículo de Educación Física del Ecuador. Guayaquil, Guayas, Ecuador: Módulo de Maestría en Formación de Profesorado Secundaria y Bachillerato, Especialidad Educación Física. 
Definición ABC. (15 de 09 de 2015). Recreación. Obtenido de http://www.definicionabc.com/social/recreacion.php

Definición de. (14 de 09 de 2015). Definición de. Obtenido de http://definicion.de/educacion-fisica/

Delors, J. (1996). La educación encierra un tesoro, informe a la UNESCO de la Comisión Internacional sobre la educación para el siglo XXI. Recuperado el marzo de 18 de 2016, de http://uom.uib.cat/digitalAssets/221/221918_9.pdf

Departamento de Educación Física, D. y. (01 de marzo de 2014). Deportes del Buen Vivir Liceo Naval. Guayaquil, Guayas, Ecuador.

Departamento, C. L. (2016). Informe Anual 2015. Guayaquil.

Dirección Nacional de Currículo. (16 de marzo de 2016). Acuerdo Ministerial N ME -2015-00168A. Obtenido de http://educacion.gob.ec/wp-content/uploads/downloads/2015/12/ACUERDONro.-MINEDUC-ME-2015-00168.pdf

Enciclopedia Colaborativa Cubana. (15 de 09 de 2015). ECURED. Obtenido de http://www.ecured.cu/index.php/Calentamiento_para_la_actividad_f\%C3\%ADsico-deportiva

Enciclopedia Colaborativa Cubana. (15 de 09 de 2015). ECURED. Obtenido de http://www.ecured.cu/index.php/Recreaci\%C3\%B3n_F\%C3\%ADsica

Espinosa, A. (11 de marzo de 2014). Acuerdo Ministerial 0041-14. Quito: Ministerio de Educación del Ecuador.

Espinosa, A. (11 de marzo de 2014). Acuerdo Ministerial 0041-14. Obtenido de http://educacion.gob.ec/wp-content/uploads/downloads/2014/03/ACUERDO-041-14.pdf

Espinosa, A. (18 de mayo de 2015). Acuerdo Ministerial 00099-A. Quito: Ministerio de Educación.

Espinoza, A. (11 de marzo de 2014). Acuerdo Ministerial 0041-14. Quito: Ministerio de Educación.

Espinoza, A. (11 de marzo de 2014). Acuerdo Ministerial 0041-14. Quito-Ecuador: Ministerio de Educación-Registro Oficial.

Espinoza, A. (11 de marzo de 2014). Acuerdo Ministerial 0041-14. Obtenido de Ministerio de Educación: http://educacion.gob.ec/wp-content/uploads/downloads/2014/03/ACUERDO041-14.pdf

Federación Internacional de Fútbol Asociado. (3 de Marzo de 2016). FIFA. Obtenido de http://es.fifa.com/mm/Document/FootballDevelopment/Refereeing/02/36/01/11/Lawsofthega mewebES_Spanish.pdf

Fernández, J. (12 de 20 de 2015). Ef Deportes. (T. Guterman, Ed.) Recuperado el 22 de marzo de 2016, de http://www.efdeportes.com/efd212/analisis-de-las-actividades-extraescolares.htm 
Fernández, J. (20 de 12 de 2015). Ef Deportes. (T. Guterman, Ed.) Obtenido de http://www.efdeportes.com/efd212/analisis-de-las-actividades-extraescolares.htm

Flavio Perlaza, Gabriela Alarcón, Carlos Angulo, Alfredo Recalde, José Guerra. (18 de 11 de 2015). EF Deportes. Obtenido de http://www.efdeportes.com/efd196/la-educacion-fisica-en-elecuador.htm

Fundación Española del corazón. (11 de Septiembre de 2015). Beneficios de la práctica deportiva para niños. Obtenido de http://www.fundaciondelcorazon.com/ejercicio/para-ninos/988beneficios-de-la-practica-deportiva-para-ninos.html

GUTIÉRREZ, M. (28 de Junio de 2004). Revista Educación. Obtenido de http://www.revistaeducacion.mec.es/re335/re335_10.pdf

Harald Muller, Wolfgang Ritzdorf. (2009). ¡Correr! ¡saltar! ¡lanzar! Santa Fe Argentina: Bill Glad/ Meter Thompson.

Iván Flor, Cristina Gándara, Javier Revelo. (2005). Manual de Educación Física Deportes y Recreación por edades. Madrid, España: Cultural, S.A.

Juan Casto, D. B. (19 de noviembre de 2015). Educación Física y salud. Obtenido de http://www.raco.cat/index.php/ApuntsEFD/article/viewFile/280885/368549

La Asociación Internacional de Federaciones Atléticas. (08 de marzo de 2016). Reglamento de $\begin{array}{lllll}\text { competición de } & \text { IAAF } & \text { Obtenido } & \text { de }\end{array}$ file:///C:/Users/Docente/Downloads/IAAF\%20Competition\%20Rules\%2020162017,\%20in\%20force\%20from\%201\%20November\%202015.pdf

Ley del Deporte, Educación Física y Recreación. (20 de febrero de 2015). Ley del Deporte, Educación Física y Recreación. Obtenido de http://www.deporte.gob.ec/wpcontent/uploads/downloads/2015/03/Ley-del-Deporte.pdf

Liceo Naval. (s.f.). Galería. Obtenido de Mejores Deportistas: http://www.liceonaval.mil.ec/webpages/index.php?url=blanco.php\&codigo $=6 \&$ codigo $2=81$

López, G. (26 de enero de 2010). Las actividades extraescolares en los centros educativos. Recuperado el 22 de marzo de 2016, de http://www.csicsif.es/andalucia/modules/mod_ense/revista/pdf/Numero_26/GEMA\%20LOPEZ\%20GUER RERO.1.pdf

Macdonald, A. (27 de noviembre de 2014). Carta de Autorización. Guayaquil: Bachillerato Internacional.

Marcelo Tovar, Roberto Rodríguez. (Septiembre de 2012). Currículo de Educación Física para la Educación General Básica y Bachillerato 2012. (M. d. Ecuador, Ed.) Recuperado el 17 de marzo de 2016, de http://educacion.gob.ec/wpcontent/uploads/downloads/2013/09/AC_Ed_Fisica_Basica_100913.pdf 
Martínez, V. (18 de noviembre de 2015). Pruebas de salud. Guayaquil, Guayas, Ecuador: Universidad Autónoma de Madrid.

Martínez. (23 de 10 de 2015). Cuestionario de datos de salud. (M. D. Morales, Entrevistador)

Ministerio de Educación -Ministerio del Deporte. (2008). Currículo de Educación Física para la Educación Básica y Bachillerato Unificado. Quito, Pichincha, Ecuador: Ministerio de Educación -Ministerio del Deporte.

Ministerio de Educación del Ecuador. (11 de Noviembre de 2015). Malla Curricular de Bachillerato General Unificado. Obtenido de http://educacion.gob.ec/malla-curricular-bachillerato-generalunificado/

Ministerio de Educación del Ecuador. (16 de marzo de 2016). Reglamento a la Ley Orgánica de Educación Intercultural. Obtenido de http://www.educar.ec/servicios/regla_loei-4.html

Ministerio de Educación del Ecuador. (2012). Currículo de Educación Física. Quito.

Ministerio de Educación del Ecuador. (2012). Currículo de Educación Física. Quito, Pichincha, Ecuador.

Ministerio de Educación. (16 de marzo de 2016). Hacia el Plan Decenal de Educación del Ecuador 2006-2015. Obtenido de http://www.oei.es/quipu/ecuador/Plan_Decenal.pdf

Ministerio de Educación. (2012). Actualización y Fortalecimiento Curricular de Educación Física 2012. Quito.

Miranda, D. (13 de marzo de 2015). Documental sobre la historia del automovilismo profesional del Ecuador. Guayaquil, Guayas, Ecuador: UDLA- Universidad de Las Américas.

Morales, D. (24 de marzo de 2015-2016). Trabajo Fin de Máster. Guayaquil, Guayas, Ecuador: Universidad Autónoma de Madrid.

Morales, D. (29 de marzo de 2016). Trabajo de Fin de Máster. Ecuador: Universidad Autónoma de Madrid.

Muñoz, J. P. (18 de marzo de 2016). forsecuador.ec. Recuperado el 18 de marzo de 2016, de http://www.forosecuador.ec/forum/ecuador/educaci\%C3\%B3n-y-ciencia/10438-himno-aldeporte

Organización Mundial de la Salud. (s.f.). Estrategia mundial sobre régimen alimentario, actividad física y salud. Obtenido de Recomendaciones mundiales sobre la actividad física para la salud: http://www.who.int/dietphysicalactivity/factsheet_recommendations/es/

Palomar, A. (2014-2015). La Educación integral del alumnado a través de la Educación Física. Madrid, España: Universidad Autónoma de Madrid.

Proyecto De Educación Física, Deportes y Recreación. (01 de marzo de 2014). Proyecto Deportes 2014. Guayaquil, Guayas, Ecuador. 
Rojas, R. (10 de junio de 2015). Blog spot. Obtenido de Propuesta del Plan curricular de Natación con Estándares de Aprendizaje: http://jimurarobert27.blogspot.com/2015/06/propuesta-del-plancurricular-de.html

Rojas, R. (29 de marzo de 2016). Tratamiento de encuestas aplicadas a cadetes del Liceo Naval 20152016. Guayaquil, Guayas, Ecuador: Liceo Naval de Guayaquil.

Romero, G. (s.f.). Revista digital innovación y experiencias educativas. Recuperado el 22 de marzo de 2016, de csif.es/andalucia/modules/mod_ense/revista/pdf/Numero_26/GUSTAVO-

http://www.csiADOLFO_ROMERO_BAREA_01.pdf

Sáenz, E. (2012-2013). Satisfacción de la Educación Física y las actividades en el medio natural. Madrid, España: Universidad Autónoma de Madrid.

Saltos, A. (2015). Deportes del Buen Vivir. Guayaquil: Liceo Naval.

Secretaría Nacional de Planificación y Desarrollo. (2013-2017). Plan Nacional del Buen Vivir. Obtenido de http://www.buenvivir.gob.ec/objetivo-3.-mejorar-la-calidad-de-vida-de-lapoblacion

Secretaría Nacional de Planificación y Desarrollo. (2013-2017). Plan Nacional del Buen Vivir. Recuperado el 17 de marzo de $2016, \quad$ de http://documentos.senplades.gob.ec/Plan\%20Nacional\%20Buen\%20Vivir\%202013-2017.pdf

Secretaría Nacional de Planificación y Desarrollo. (22 de marzo de 2016). Plan Nacional del Buen Vivir 2013-2017. Obtenido de http://www.buenvivir.gob.ec/objetivo-4.-fortalecer-lascapacidades-y-potencialidades-de-la-ciudadania

UNESCO. (16 de marzo de 2016). Carta Internacional de la Educación Física y el Deporte. Obtenido de http://portal.unesco.org/es/ev.phpURL_ID=13150\&URL_DO=DO_TOPIC\&URL_SECTION=201.html

UNESCO. (18 de 11 de 2015). UNESCO.ORG. Obtenido de http://portal.unesco.org/es/ev.phpURL_ID=13150\&URL_DO=DO_TOPIC\&URL_SECTION=201.html

UNESCO. (2015). Educación Física de calidad-Guía para los responsables políticos. Obtenido de http://unesdoc.unesco.org/images/0023/002313/231340s.pdf

UNESCO. (21 de noviembre de 1978). Carta Internacional de la Educación Física y el Deporte. Obtenido de http://portal.unesco.org/es/ev.phpURL_ID=13150\&URL_DO=DO_TOPIC\&URL_SECTION=201.html

Universidad Interamericana de Puerto Rico. (27 de 10 de 2015). Ciencias del Movimiento humano y la salud. Obtenido de http://www.saludmed.com/rxejercicio/rxejercicio.html

Web consultas. (14 de 09 de 2015). Obtenido de http://www.webconsultas.com/ejercicio-ydeporte/vida-activa/beneficios-del-ejercicio-fisico-869 